\title{
Es fehlt am Geld, nicht an den Leuten
}

Wie stellen wir es an, damit die Schweiz in Zukunft nicht nur genügend, sondern auch die «richtigen» Ärztinnen und Ärzte ausbildet? Diese Frage stand am Anfang der SÄZ-Podiumsdiskussion in Basel, und sie führte zu einer animierten Diskussion. Für alle Beteiligten war klar: Der «Import» von ausländischen Ärzten ist nicht das Modell der Zukunft. Nach Alternativen wird fieberhaft gesucht.

Anna Sax

anna.sax@saez.ch
«Unterhaltung, intellektuelle Befriedigung und hoffentlich auch neue Ideen» - so umschreibt der Moderator Werner Bauer zu Beginn der Diskussion die Ziele des Podiums. Und er schickt den Wunsch nach, die guten Ideen seien dann in der Ärztezeitung zu veröffentlichen, was Chefredaktor Bruno Kesseli gerne zusagt. Zunächst aber führt Bauer anhand von Zahlen nochmals den «Mangelkomplex» vor Augen, mit dem wir es zu tun haben. Nur noch gut die Hälfte der in der Schweiz tätigen Ärztinnen und Ärzte haben heute ein schweizerisches Staatsexamen in der Tasche. 2009 wanderten über 1300 ausgebildete Ärzte aus dem Ausland ein, während an den Schweizer Universitäten nur 728 ihren Abschluss machten. 29\% aller Weiterbildungstitel gehen an ausländische Kolleginnen und Kollegen. Er schliesst seine kurze Präsentation mit vier Thesen: 1. Es besteht ein Mangel an Ärzten, die in der Schweiz ausgebildet worden sind. 2. Das Füllen der Lücke mit Kollegen aus dem Ausland ist keine nachhaltige Lösung. 3. An einer Vermehrung der Studienplätze führt kein Weg vorbei. 4. Die Verwesentlichung der ärztlichen Arbeit und die
Förderung interprofessioneller Strukturen könnten einen Beitrag zur Lösung leisten.

Für Moderator und Podiumsteilnehmende ist klar, dass es nicht darum geht, einheimische gegen ausländische Kräfte in den Spitälern auszuspielen. Für die Patientinnen und Patienten spiele die Herkunft des Arztes nämlich keine Rolle, sind sie sich einig. Das Problem liegt vielmehr darin, dass das Gesundheitspersonal, das die Schweizer Spitäler aus dem Ausland rekrutieren, anderswo fehlt.

\section{Der Bund ist mitverantwortlich}

Die Verantwortung für die Ausbildung der Ärztinnen und Ärzte liegt bei den Kantonen, und so muss sich der basel-städtische Gesundheitsdirektor Carlo Conti als Erstes die Frage stellen lassen, ob die Kantone versagt hätten. Dieser nimmt den Ball im wahrsten Sinne des Wortes und in typisch baslerischer Manier auf, indem er auf den hiesigen Fussballclub verweist: «Wieso ist der FC Basel seit Jahren an der Spitze des Schweizer Fussballs?», fragt er, um gleich die Antwort nachzuliefern: «Weil er viel in die Jugendförderung

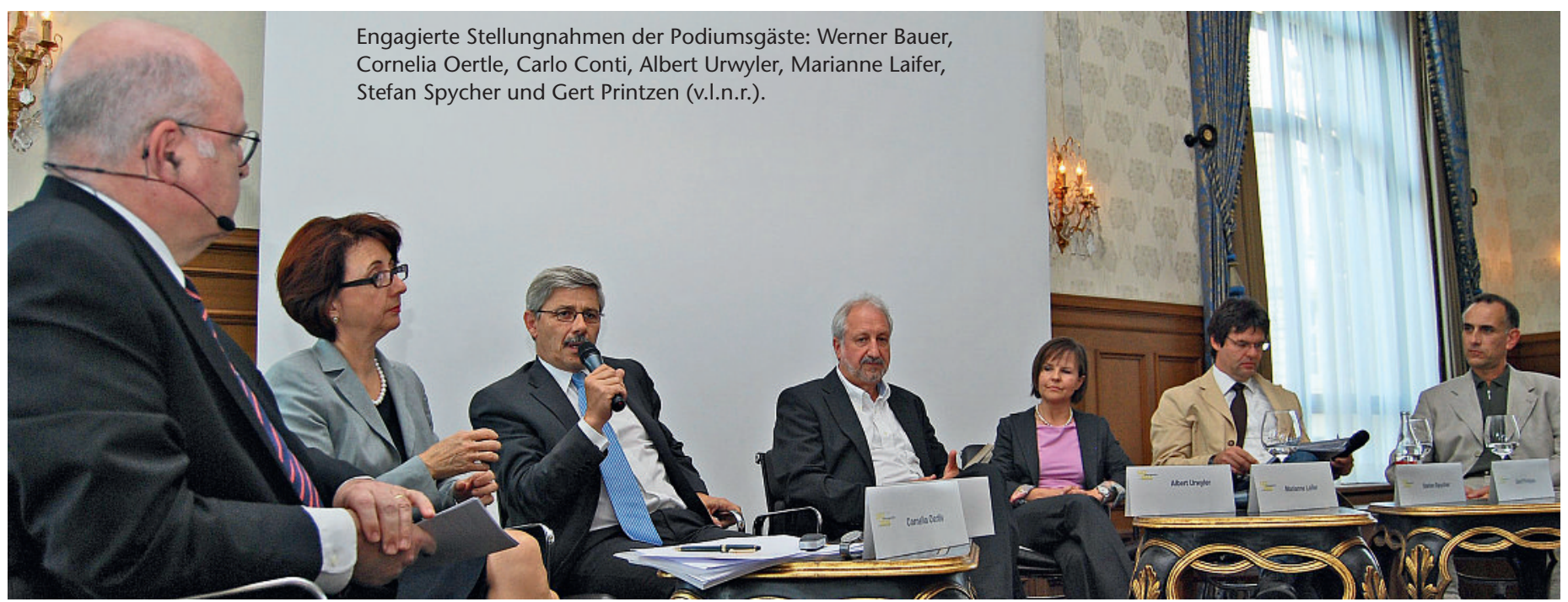


investiert hat.» Weil wir zu wenig in den akademischen Nachwuchs investierten, werde es zunehmend schwierig, Fachleute für die Spitäler zu finden, fährt Conti fort. Verantwortlich dafür seien einerseits die Leistungserbringer - vor allem bei der Pflege - und andererseits die Kantone. So versucht der Gesundheitsdirektor nicht, sich aus der Pflicht zu stehlen, doch sieht er sich auch bis zu einem gewissen Grad machtlos: «Es fehlt an den nötigen Finanzen. Diese müssen aus den Bildungs- und nicht aus den Gesundheitsbudgets der Kantone bereitgestellt werden», stellt Conti klar.

\section{«Es ist nicht fair, im grossen Stil}

Gesundheitspersonal anzuwerben, dlas anderswo ausgebildet worden

ist.» Stefan Spycher

So könne sich also der Bund gemütlich zurücklehnen, wendet sich der Gesprächsleiter an den Vizedirektor des Bundesamtes für Gesundheit BAG, Stephan Spycher. Dieser lässt sich ohne weiteres aus der Reserve locken: «Wir sind für die Gesundheitspolitik mitverantwortlich», bestätigt er. Natürlich liege die Hauptverantwortung für die Ausbildung der Ärztinnen und Ärzte bei den Universitäten und damit bei den Kantonen, doch der Bund wirke dort, wo er könne. Zum Beispiel in der Gesundheits-Aussenpolitik: «Der WHO-Code verpflichtet uns zu fairem Verhalten. Und es ist nicht fair, im grossen Stil Gesundheitspersonal anzuwerben, das anderswo ausgebildet worden ist.» Wie genau der Bund zu mehr Fairness beitragen will, bleibt jedoch offen. Jedenfalls sind die von Gesprächsleiter Bauer ins Spiel gebrachten

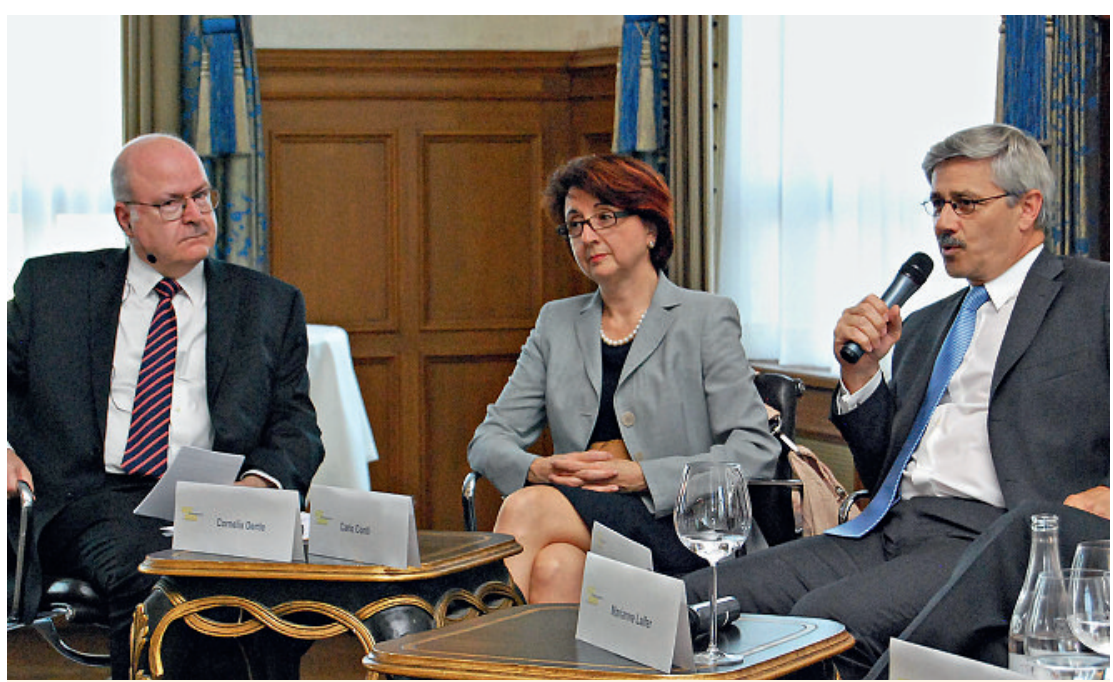

Fussball als Vorbild: Wie der FC Basel, so müsse auch das Gesundheitswesen den Nachwuchs fördern, findet der Vorsteher des Gesundheitsdepartements Basel-Stadt Carlo Conti.
Schweizer Stipendien für Medizinstudierende an der Budapester Semmelweis-Universität keine Option für Spycher. Lieber will er die Zügel selber in der Hand behalten: «Bei der medizinischen Ausbildung bieten wir eine sehr gute Qualität.»

Es fehlt, wie mehrere Votanten auf dem Podium und im Publikum betonen, nicht an Kandidatinnen und Kandidaten für das Medizinstudium, sondern an Studienplätzen und an Geld. Da liegt die Frage an den Dekan der medizinischen Fakultät der Universität Basel nahe, ob substantielle Kapazitätssteigerungen aus Sicht der Universitäten überhaupt möglich wären - vorausgesetzt, die notwendigen Ressourcen würden bereitgestellt. Der angesprochene Albert Urwyler relativiert: «Geld ist das eine. Man könnte eine solche Übung stemmen, aber es wäre ein grösserer Effort notwendig, gerade auch im organisatorischen Bereich.» Es brauche Räume, denn ein Medizinstudium im Schichtbetrieb sei kaum möglich, führt Urwyler weiter aus. Bis eine Kapazitätserweiterung Wirkung zeitige, brauche es 10 Jahre.

\section{«Es wäre ein grösserer Effort notwendig, gerade auch im orga- \\ nisatorischen Bereich.» Albert Urwyler}

\section{Akademikerinnen im Vormarsch}

Bis hierher ist die Ausbeute an guten Ideen für die SÄZ-Leserinnen und -leser noch nicht berauschend: Die Kantone haben kein Geld, der Bund schaut zu, die Universitäten brauchen Zeit. Bauers Vorschlag für Semmelweis-Stipendien hat kein Echo gefunden. So klopft er mit einer weiteren Idee auf den Busch, die schon seit längerem immer wieder auftaucht: Sollten Ärztinnen und Ärzte nicht von Arbeiten entlastet werden, die andere ebenso gut machen können? Warum sollen Echokardiographien oder Endoskopien nicht von fachlich qualifiziertem Pflegepersonal gemacht werden, wie es in anderen Ländern bereits der Fall ist? Cornelia Oertle, Leiterin des Fachbereichs Gesundheit der Berner Fachhochschule, gibt sich zunächst reserviert: «Ob wir helfen können, wird sich weisen. Wir bilden an den Fachhochschulen Pflegende aus, die sicher gewisse Aufgaben übernehmen können - aber wir haben ja auch Mangel an Pflegepersonal.» Oertle war von Anfang an beteiligt an den Ausbildungsreformen für Pflegende. Ziel dieser Reformen sei es nie gewesen, den Aufgabenbereich in Richtung ärztliche Tätigkeit auszuweiten, sondern den Bedarf an gut ausgebildeten Pflegenden im stationären Bereich zu decken, habe es doch während langer Jahre keine entsprechenden Bildungsmöglichkeiten in der Schweiz gegeben. "Allerdings hat die Diskussion über neue Versorgungsmodelle in letzter Zeit an Intensität gewonnen. Ärztenetzwerke zeigen vermehrt 
Interesse an einer Zusammenarbeit mit Pflegefachpersonen», stellt Oertle fest. Obwohl sie noch viele Haken ortet, zum Beispiel bei der Abrechnung, sieht sie die Chancen dieser Entwicklung: «Mit Bachelorund Mastertiteln, mit neuen Berufsfeldern wie Advanced Nurse Practitioners, werden auch Akademikerinnen angesprochen, die umsteigen möchten und sonst den Pflegeberuf vielleicht nicht gewählt hätten.»

«Wir bilden an den Fachhochschulen Pflegende aus, die sicher gewisse Aufgaben übernehmen können - aber wir haben ja auch Mangel an Pflegepersonal.» Cornelia Oertle

Angesprochen fühlen sich Akademikerinnen jedenfalls vom Beruf der Ärztin. Die Frauenquote bei den Staatsexamen liegt bereits bei über $60 \%$. Fragt sich nun, ob das eher eine Chance oder einen Nachteil darstellt für die ärztliche Versorgung, denn Frauen haben in der Regel tatsächlich einen anderen Zugang zum Arztberuf als Männer, wie Marianne Laifer, CoPräsidentin von Medical Women Switzerland, ausführt: «Ärztinnen arbeiten anders. Sie verbringen mehr Zeit mit den Patientinnen und Patienten, und sie wählen weniger technische Sparten der Medizin.» Der Ärztemangel werde dadurch in gewissen Gebieten wie etwa der Chirurgie verstärkt; in anderen Bereichen wie Gynäkologie oder Pädiatrie, wo zunehmend Frauen tätig seien, sinke das Prestige, wie bereits in anderen Branchen mit steigendem Frauenanteil beobachtet, benennt Laifer das Problem unumwunden. Weil geregelte Arbeitszeiten und Möglichkeiten zur Teilzeitarbeit für Ärztinnen spätestens dann zum Thema werden, wenn die Kinderfrage ins

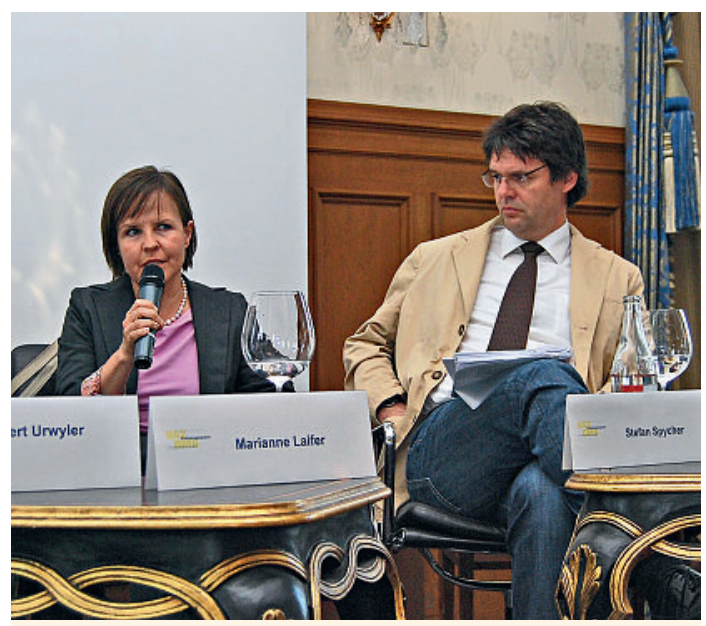

Warum Ärztinnen Gruppenpraxen und städtische Agglomerationen als Arbeitsort bevorzugen, erklärt Marianne Laifer, Co-Präsidentin von Medical Women Switzerland.

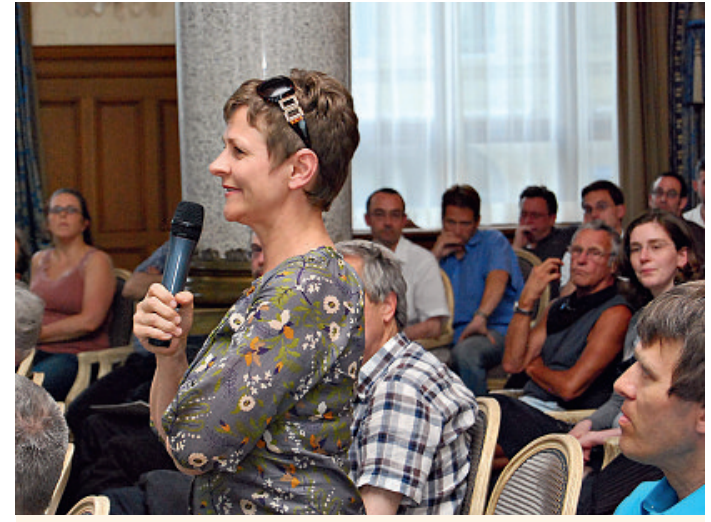

So lange Hausärzte weniger verdienen als Spezialisten, sei es schwer, Interessenten zu finden, meint die Präsidentin der Vereinigung der Hausärzte beider Basel, Eva Kaiser.

Spiel kommt, bevorzugen sie Gruppenpraxen und städtische Agglomerationen als Arbeitsort. Oder aber «sie verschwinden in Richtung Pharmaindustrie oder Telemedizin», wie eine Chirurgin aus dem Publikum einwirft. «Die Segregation findet nicht nur in horizontaler, sondern vor allem auch in vertikaler Richtung statt», fährt Laifer weiter: «Auf professoraler Ebene liegt der Frauenanteil in der Medizin bei gerade noch $14 \%$.»

\section{«Ärztinnen arbeiten anders. Sie verbringen mehr Zeit mit den \\ Patientinnen und Patienten, und \\ sie wählen weniger technische \\ Sparten der Medizin.» Marianne Laifer}

\section{Es braucht Hausärzte, nicht Schönheits- chirurgen}

Die Ärztinnen und Ärzte seien heute dem Arbeitsgesetz unterstellt und die Weiterbildungsmöglichkeiten hätten sich verbessert, räumt Gert Printzen als Vertreter der angestellten Ärzte im Zentralvorstand des FMH ein. Dennoch sieht er Optimierungspotential, etwa indem Assistenzärztinnen von administrativen Arbeiten entlastet würden: «Die ärztlichen Kernaufgaben gilt es zu fördern, damit kann der Arztberuf nochmals deutlich attraktiver werden.»

An dieser Stelle schaltet sich aus dem Publikum Dr. René Salzberg ein, der frühere Leiter der Aus-, Weiter- und Fortbildung im FMH Zentralvorstand: Man könne ja einmal damit anfangen, die Studentenzahl zu erhöhen, anstatt immer nur darüber zu reden, was es alles an Vorbereitung brauche, bemerkt er trocken, was wiederum Gesundheitsdirektor Conti auf den Plan ruft: «Also wovon reden wir jetzt eigentlich? Vom Nachwuchs oder vom System?» An Interessierten, die Medizin studieren wollten, fehle es nicht, 
wiederholt Conti. Es brauche nicht nur mehr Geld, sondern zum Beispiel auch die Bereitschaft der Universitäten, Mittel zu verschieben: «Wo arbeiten all die Juristen und Medienwissenschaftlerinnen, die an der Universität ausgebildet werden?» Doch die Zahl der

«lch bin nicht bereit, 100 zusätzliche Studienplätze zu finanzieren, damit wir am Schluss 100 neue Schönheitschirurgen haben.» Carlo Conti

ausgebildeten Ärztinnen und Ärzte sagt noch nicht alles aus: «Ich bin nicht bereit, 100 zusätzliche Studienplätze zu finanzieren, damit wir am Schluss 100 neue Schönheitschirurgen haben», macht Conti klar, «wir brauchen vor allem Hausärzte.»

\section{Das eine tun und das andere nicht lassen}

Damit ist die Diskussion richtig lanciert: Müssen sich die Studierenden früher auf eine FMH-Richtung festlegen? Ist die Einzelpraxis wirklich von gestern? Was tun die Spitäler, um Frauen eine medizinische Karriere zu ermöglichen? Zu all diesen Fragen wird unter Beteiligung des Publikums lebhaft debattiert; die Meinungen über den besten Weg zur optimalen Gesundheitsversorgung sind offensichtlich geteilt. Die Forderung, die Hausarztmedizin sei aufzuwerten, wird mehrfach erhoben. «Bitte lassen Sie uns heute Abend nicht auch noch über die Hausarztinitiative diskutieren», interveniert der Gesprächsleiter an dem Punkt,

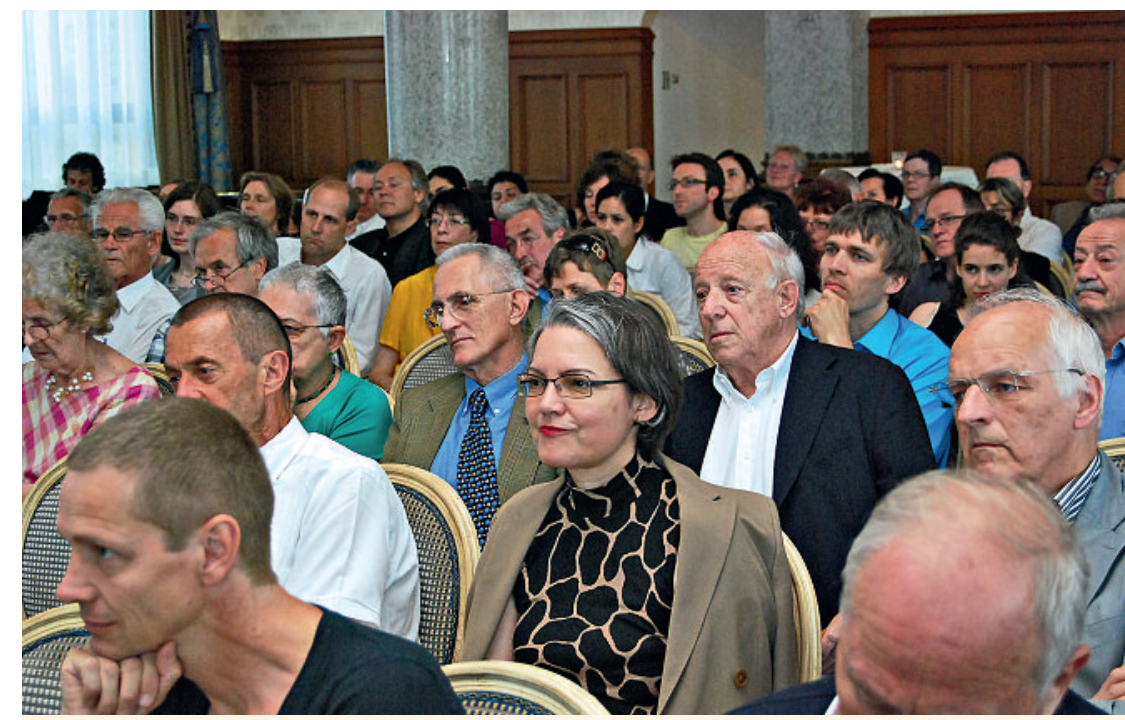

Zahlreich erschienen und engagiert bei der Diskussion: das Publikum der SÄZ-Podiumsdiskussion.

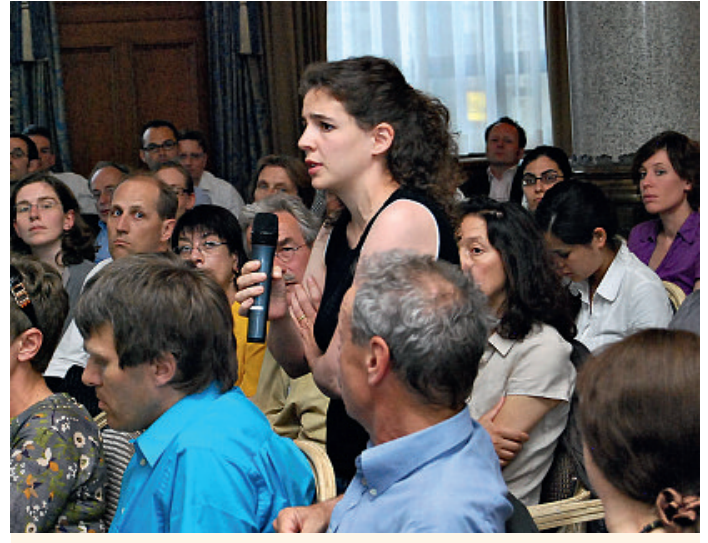

Auch die Präsidentin des Forums Junger Chirurgen, Bettina Wölnerhannsen, zeigte sich besorgt wegen des fehlenden Nachwuchses in der Schweiz.

wo Spycher dazu ansetzt, den Gegenentwurf des Bundes zu begründen. Printzen fordert die Aufhebung des Zulassungstopps für alternative Modelle wie Gruppenpraxen. Laifer will transparentere Strukturen für die Talentförderung wie z.B. Mentoring-Programme, was es laut Urwyler zumindest in Basel längst gibt. In dieser Phase zeigt sich, dass es an Ideen nicht fehlt, diese jedoch aus Systemgründen nicht einfach umzusetzen sind. Oertle warnt davor, in einen «Riesenaktivismus» zu verfallen. Neue, berufsübergreifende Versorgungsmodelle bräuchten Zeit, Pilotprojekte sollten Klarheit bringen. «Damit integrierte Versorgung gelingt, braucht sie Akzeptanz in der Bevölkerung und in Ärztekreisen.»

\section{«Die ärztlichen Kernaufgaben gillt es zu fördern, damit kann der Arztberuf nochmals deutlich} attraktiver werden.» Gert Printzen

Es bleibt dabei: Die von Bauer erhoffte «neue, interdisziplinäre, revolutionäre Idee» wird auch an diesem Abend nicht geboren. Was dagegen herüberkommt, ist der Eindruck, dass die verschiedenen Player mehr als auch schon bereit sind, einander zuzuhören und gemeinsam nach Lösungen zu suchen. «Das eine tun und das andere nicht lassen», so könnte man das Fazit dieser Veranstaltung zusammenfassen: Wir müssen den Nachwuchs fördern und zugleich über neue Modelle der Arbeitsteilung nachdenken.

Übrigens: Der FC Basel wird am Tag darauf Schweizer Meister. 